\title{
THz Wave Propagation on Strip Lines: Devices, Properties, and Applications
}

\author{
Yutaka KADOYA, Masayuki ONUMA, Shinji YANAGI, Tetsuya OHKUBO, \\ Naoko SATO, Jiro KITAGAWA \\ Dept. of Quantum Matter, Hiroshima University, Higashihiroshima, 739-8530, Japan \\ kd@hiroshima-u.ac.jp
}

\begin{abstract}
We report the propagation characteristics of THz pulses on micro-strip-lines and coplanar strip-lines, in which low permittivity polymer materials are used as the dielectric layer or the substrate. As a result of the low attenuation and small dispersion in the devices, the spectral width up to $3 \mathrm{THz}$ can be achieved even after the $1 \mathrm{~mm}$ propagation. Spectroscopic characterizations of liquid or powder specimens are demonstrated using the devices. We also show a possibility of realizing a very low attenuation using a quadrupole mode in three strip coplanar lines on the polymer substrate.
\end{abstract}

\section{Keywords}

Terahertz, strip-line, time-domain spectroscopy.

\section{Introduction}

Time domain spectroscopy (TDS) is one of the important applications of $\mathrm{THz}$ electromagnetic waves generated and detected using femtosecond (fs) laser pulses. Though the present $\mathrm{THz}$ TDS systems utilizing $\mathrm{THz}$ waves propagating in free space work quite well, it is desired to integrate it in a solid state chip to make the system more compact and functional. Waveguides suitable for such high frequency electromagnetic waves are the printed strip lines such as micro-strip-lines (MSLs), and coplanar-strip-line (CPSs).

Generation of ultrafast electrical pulses by fs laser pulses in semiconductor strip-lines has a history since 80's [1-5]. Though MSL structures were considered originally [2], [3], the efforts soon shifted to CPSs, mostly because of the easiness of fabrication keeping the quasi-single mode propagation and the characteristic impedance close to $50 \Omega$ [4], [5]. Then, numerous investigations have been carried out to characterize and improve the performance of the CPSs. A spectroscopy of powder specimen was also demonstrated, though the frequency was limited below $1 \mathrm{THz}$ [6]. In most of the experiments, the CPSs were formed on ion-dosed silicon or low-temperature-grown (LTG) GaAs substrates, which themselves serve as the photoconductive (PC) material having sub-ps response time used for the generation and detection of $\mathrm{THz}$ pulses. Semiconductor-based CPSs, however, suffer from a large attenuation and dispersion. The dominant loss mechanism in $\mathrm{THz}$ range is the radiation of energy as a shock wave [7]. Since they come from the difference in the permittivity between the substrate and the air, it is desired to use low permittivity substrate. Though a silica-based membrane was presented in the previous work [8], it is not simple in fabrication and not very robust mechanically. On the other hand, in 1997, a low loss and small dispersion propagation was reported using the MSLs with $10 \mu \mathrm{m}$-thickness dielectric layer based on low permittivity polymer materials [9]. It was also demonstrated that the MSLs can be used as a sensitive label-free detector of DNA molecules, by using a resonator structure in the MSL, though the spectral range of the devices was still in sub-THz range [10].

In this paper we report the propagation characteristics of THz pulses on MSLs and CPSs, investigated computationally and experimentally. In particular, we show that, by the use of low-permittivity dielectric materials, they can support the propagation of electrical pulses whose spectra reaching $3 \mathrm{THz}$ for a distance as long as $1 \mathrm{~mm}$. As an application of these devices, also we demonstrate the $\mathrm{THz}$ spectroscopic characterization of liquid and powder specimens [11-13]. In addition, we show that even lower attenuation can be realized in quadrupole mode in threestrip CPS (TSCPS) on low permittivity substrate.

\section{Devices, Measurement, and Simulation}

\subsection{Device Structures and Fabrication Procedures}

Fig. 1(a) shows the cross-sectional birds-eye view of the MSL devices [11]. On an Au-deposited glass substrate, a polyimide film (TORAY; SP483, $\varepsilon \sim 3.2$ ) of 10 20 $\mu \mathrm{m}$ thickness was formed by spin-coating and curing the precursor. On the dielectric, a piece of LTG-GaAs of $800 \mathrm{~nm}$ thickness was Van der Walls bonded, and mesa 
etched to $40 \times 100 \mu \mathrm{m}^{2}$ rectangles used as the PC switches for the generation and detection of $\mathrm{THz}$ pulses. The distance between the generation and the detection PC switches is $1 \mathrm{~mm}$. Then a $20 \mu \mathrm{m}$ wide and $200 \mathrm{~nm}$ thick Au line was defined by a conventional lift-off technique to form a MSL structure, with biasing and probing lines at the positions of the PC switches. To enhance the adhesion, a $5 \mathrm{~nm}$ thick Ti was inserted between the polyimide and $\mathrm{Au}$. One of the ends of the line is electrically opened so as to serve as a reflector used for the evaluation of the propagation constants. In the devices used in the measurement of liquids, the line was covered by an additional polyimide layer of $10 \mu \mathrm{m}$ thickness and a vessel made of polyethylene was formed on it. Also, for the optical excitation of the PC switches from the substrate side, small holes were prepared in the Au ground plane at the position of the PC switches. The dielectric constant and the loss of the polyimide film were measured with a conventional THz TDS system and shown in Fig. 2(a). For the measurement, a polyimide film of about $300 \mu \mathrm{m}$ thickness was separately prepared simply by dipping the precursor to a frame and curing it. Hence, the quality of the film could be different from that used in the MSL devices. However, as shown later, the experimental results are consistently explained.
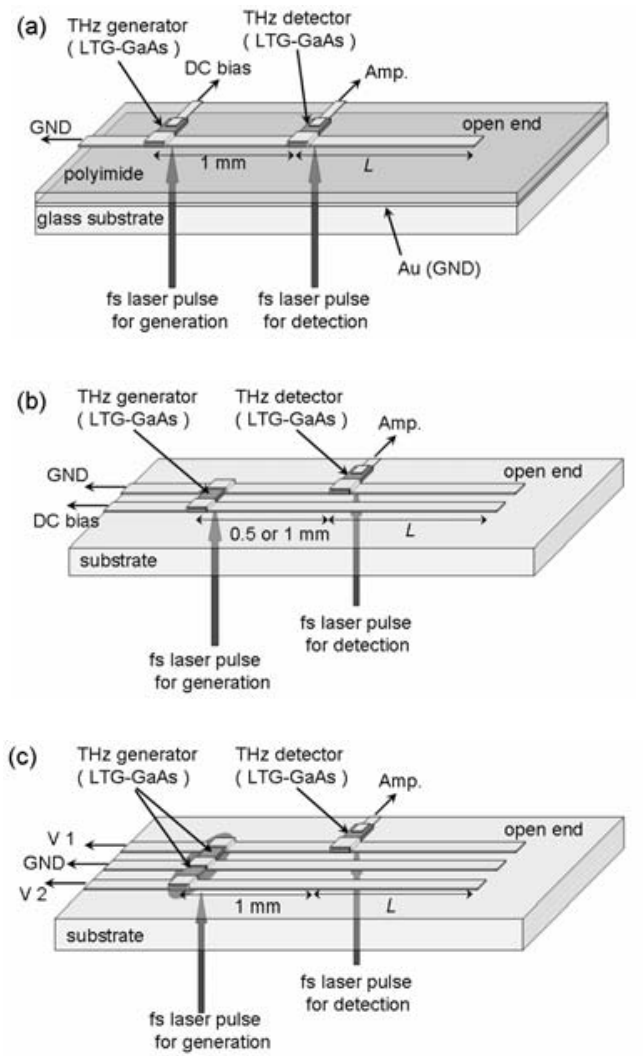

Fig. 1. Structure of the MSL (a), CPS (b), and TSCPS (c) devices used in this work.

The fabrication of CPSs or TSCPSs is even simpler [13]. On a dielectric substrate, a pallarel-line pattern made of $\mathrm{Ti} / \mathrm{Au}$ (same thickness as MSLs) was fabricated with PC switches made of LTG GaAs in the same way as the MSLs. The CPS and TSCPS patterns used in this work are de- picted in Fig. 1(b) and 1(c), respectively. Both the line width and the space between the lines are $20 \mu \mathrm{m}$. As the low permittivity substrates, we used commercial polyer plates (PAX; Tsurupica, or ZEON; ZEONEX, $\varepsilon \sim 2.3$ ). In our experiments, no difference was found between the two products. We also tested the CPSs and TSCPSs made on quartz and sapphire substrates for comparison. In the sapphire CPS, the distance between the generation and the detection CPS is $0.5 \mathrm{~mm}$ and that in the quartz and polymer CPSs is $1 \mathrm{~mm}$. The properties of the substrates are shown in Fig. 2(b).
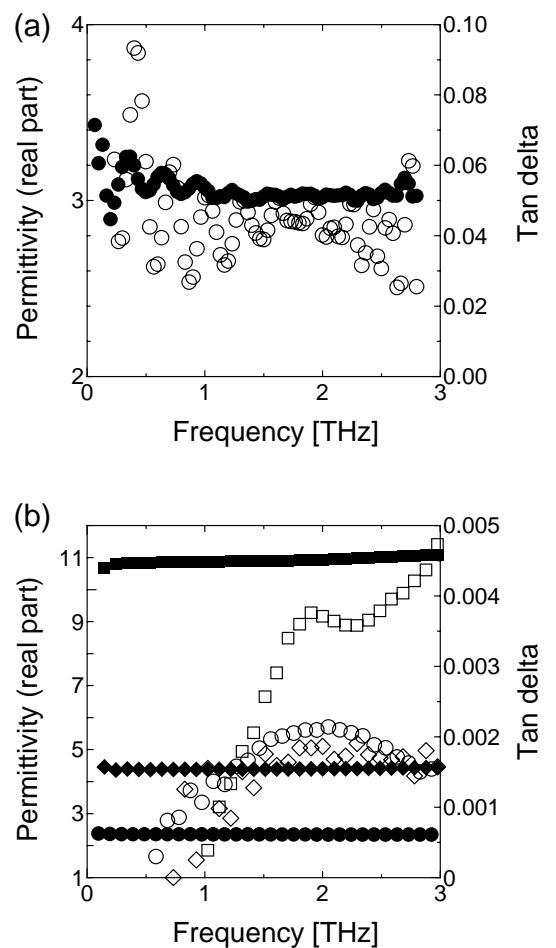

Fig. 2. Properties of the (a) polyimide film and (b) substrates used in this work. The filled and open symbols are the real part of the permittivity and $\tan \delta$, respectively. In (b), the squares, diamonds, and circles correspond to the sapphire, quartz, and polymer substrates, respectively.

\subsection{Measurement and Simulation}

The setup for the measurement of $\mathrm{THz}$ pulses on the strip-lines is basically the same as that of conventional $\mathrm{THz}$ TDS systems. The $\mathrm{THz}$ pulses were generated by exciting the dc-biased PC switch with fs optical pulses, which was delivered from a mode-locked Ti-Sapphire laser. The pulse width, the center wavelength, and the repetition rate are about $200 \mathrm{fs}, 800 \mathrm{~nm}$, and $76 \mathrm{MHz}$, respectively. The signals propagating along the lines were lock-in detected as the average current in the detector PC switch, which was excited by the laser pulses with various delay time from the $\mathrm{THz}$ generation. A fiber laser whose pulse width is about 60 fs was also used as the light source with a second harmonic generator. The experimental results shown below were obtained using the Ti-Sapphire laser, unless otherwise specified. 
The propagation constants (attenuation and effective index of refraction) were evaluated experimentally in the following way [13]. A $\mathrm{THz}$ pulse generated at the $\mathrm{PC}$ switch propagates along the line, passes by the detector, and arrives at the open end, where it is reflected and propagates backward passing by the detector again. Hence we can observe the pulse twice. From the two peaks curved out from the time-domain trace the amplitude and the phase spectra were calculated by the Fourier transformation. Then we can evaluate the change of the spectra due to the propagation and reflection. Using such spectra obtained with the devices of different length $L$ between the detector and the open end, we can eliminate the effect of the reflection and calculate the propagation constants, as far as the reflection properties are not different between the devices of the same type, which may be justified as they are determined by the well-controlled lithographic process. In this method, possible variations of the generation and detection characteristics can be canceled out.

The propagation of $\mathrm{THz}$ pulses was simulated using a finite-difference time-domain (FDTD) software (Mizuho $\mathrm{RI}$; Emerge). In the simulation, the conductance of Au was assumed to be $4.5 \times 10^{7} \Omega^{-1} \mathrm{~m}^{-1}$, which is close to the measured value, $\sim 3 \times 10^{7} \Omega^{-1} \mathrm{~m}^{-1}$ of a film deposited on the polymer. The polymer, quartz, and sapphire substrates and the polyimide film were assumed to have a constant permittivity, 2.3, 4.5, 10.8, and 3.2, respectively. They were also assumed to be loss-free so that the dielectric loss was not considered in the simulation.

\section{Micro-strip Lines}

\subsection{Propagation Characteristics}

Shown in Fig. 3 is an example of the time domain traces observed in the MSL device of $L=2 \mathrm{~mm}$. In this experiment, the fiber laser was used as the light source. As mentioned above, the pulse was detected twice. Note that the two peaks are well resolved, indicating the small distortion during the $4 \mathrm{~mm}$-propagation and reflection. In this particular case, two peaks were carved out from the trace as the one from 0 to 35 ps for the first peak and that from 35 to 70 ps for the second peak. Depicted in the inset is the amplitude spectrum of the first pulse, demonstrating that the spectral range reaches $3 \mathrm{THz}$. The attenuation constant and the effective index of refraction were evaluated using the devices of $L=1$ and $2 \mathrm{~mm}$, and plotted as functions of frequency by the filled circles in Fig. 4. In the figures, the results of the FDTD simulation were also plotted by the open squares.

The attenuation evaluated experimentally increases with frequency and exceeds $1 \mathrm{~mm}^{-1}$ at $1.2 \mathrm{THz}$. The experimental values are obviously higher than those of the FDTD simulation and the deviation increases with frequency. Shown by the solid line is the prediction of the attenuation due to the conductor loss based on the analytical formula used in microwave regime [8], [14]. The formula reproduces well the FDTD result. Hence, as a possible cause of the additional attenuation, we estimated the attenuation due to the dielectric loss also with a formula used in microwave regime [14] using the values of $\tan \delta$ shown in Fig. 2(a). The results were plotted by the open circles. Though the estimated values undulate due to the influence of the interference in the measurement of $\tan \delta$, they can account for the experimental results. Hence, the additional attenuation is most likely to stem from the dielectric loss of the polyimide film. However, further investigation may be necessary since the variation of polymer thickness can also cause the attenuation.

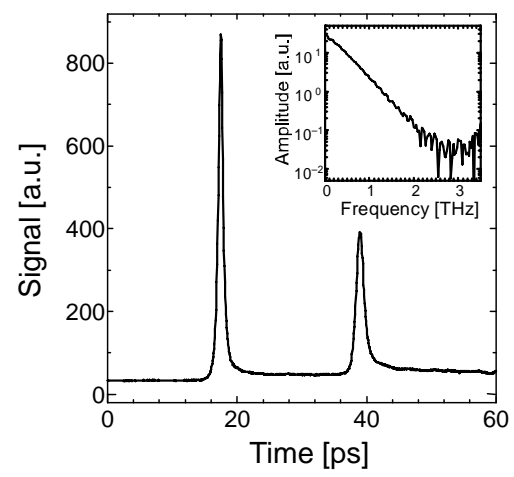

Fig. 3 An example of the time-domain trace observed in the MSL device of $L=2 \mathrm{~mm}$. The inset shows the amplitude spectrum of the first peak.
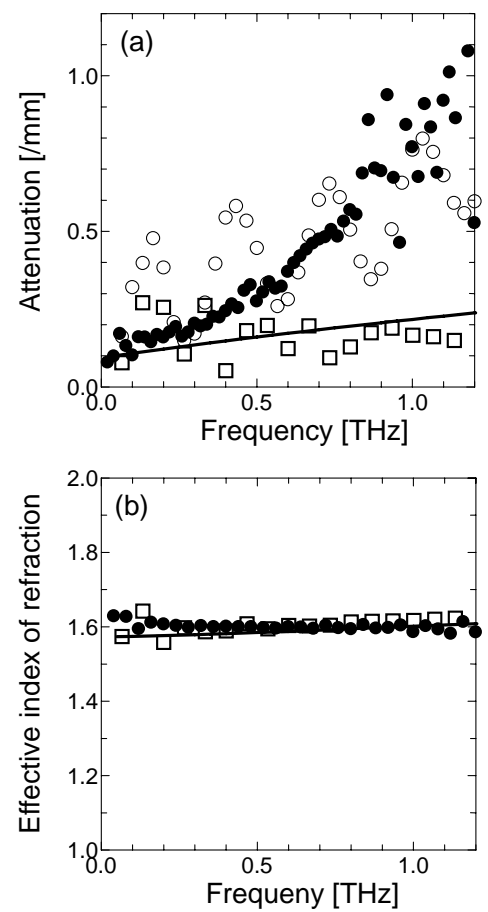

Fig. 4 (a) Attenuation constant and (b) effective index of refraction associated with the propagation of the $\mathrm{THz}$ pulses along the MSL. The filled circles and open squares represent the experimental and FDTD results, respectively. The solid line and the open circles in (a) are the values estimated using the analytical expressions used in microwave regime with the measured conductance of the metal line and the $\tan \delta$ of the polyimide film. The solid line in (b) is the prediction based on the model used in microwave regime. 
In contrast, the experimental result of the effective index of refraction is consistent with the FDTD simulation. The slight decrease with frequency in the experimental result comes from that in the dielectric constant shown in Fig. 2(a). It should be noted that the dispersion is very small in the range up to $1.2 \mathrm{THz}$. A formula for the dispersion used in microwave regime [15] was also compared as shown by the solid line and found to reproduce well the experimental and FDTD results within the frequency range shown here. However, though not shown here, we also found that a small but apparent deviation from the FDTD values shows up in the range higher than $1 \mathrm{THz}$.

\subsection{Spectroscopy of Liquid Specimen}

One of the weak points in the THz TDS is the large attenuation of the $\mathrm{THz}$ waves in polar liquids, while the important phenomena such as the libration of liquid molecules and the hydration dynamics in aqueous solution appear in $\mathrm{THz}$ regime. As one of the way to solve the problem, we tested the use of the MSL device for the spectroscopy of polar liquids [11]. For such purpose, to control the interaction between the $\mathrm{THz}$ waves and the specimen, we used the MSL in which the signal line was covered by a $10 \mu \mathrm{m}$-thick spacer layer.

Depicted in Fig. 5(a) are the time domain traces and the amplitude spectra of the signals with and without water on the MSL. The narrower spectral width in comparison with Fig. 3 is likely to be due to the difference of the pulse width of the laser. In Fig. 5(b), the attenuation constant of the water was plotted as a function of frequency. Since the attenuation constant of the bulk specimen can not be measured directly in the MSL, the values were converted through a comparison with the results obtained in an attenuated total reflection (ATR) method. Details of the conversion procedure have been reported elsewhere [16]. In the figure, reported values [17] were also plotted for comparison. The good correspondence suggests merely that the ATR measurement was successful. However, an important fact is that, in the MSL, the attenuation can be measured continuously from microwave to $\mathrm{THz}$ range, without the discontinuity seen around $0.5 \mathrm{THz}$ in the report which merges the results obtained in the separate experiments. In addition, it is possible to extend the present device as a remote sensor head by using optical fibers for the delivery of excitation pulses, enabling, for example, a measurement in a liquid.

Shown in Fig. 6 is the change in the attenuation with the immersion of bovine-serum-albumin (BSA) molecules at various densities [12]. With the increase of the density, the attenuation decreases because of the capturing of the free water molecules due to the hydration of albumin molecules. The change is almost linear with density. Further analysis may give us an insight into the dynamics of the hydration.
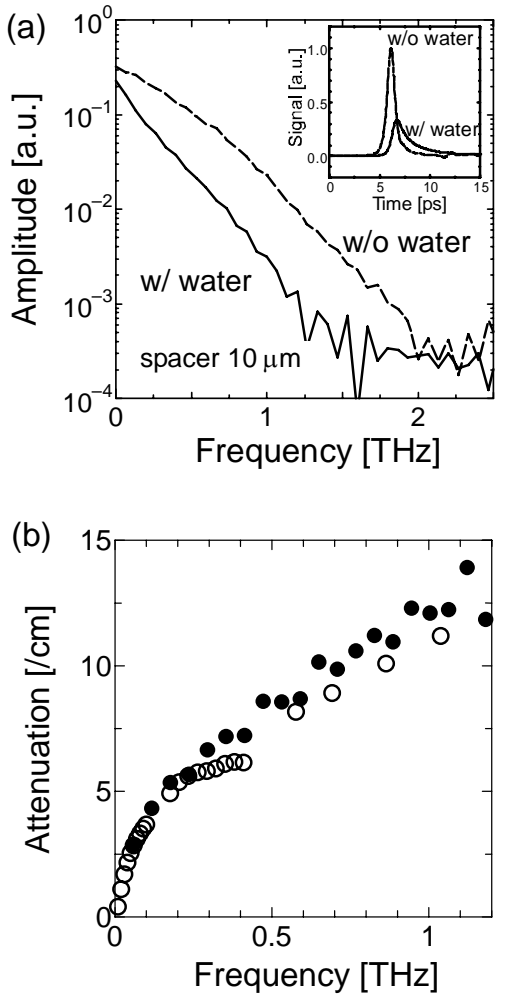

Fig. 5 (a) Amplitude spectra with and without water on the MSL. The inset shows the observed time-domain traces. (b) The filled circles are the attenuation constant of water evaluated from the spectra shown in (a) after a conversion to the bulk values. The open circles are the reported values.

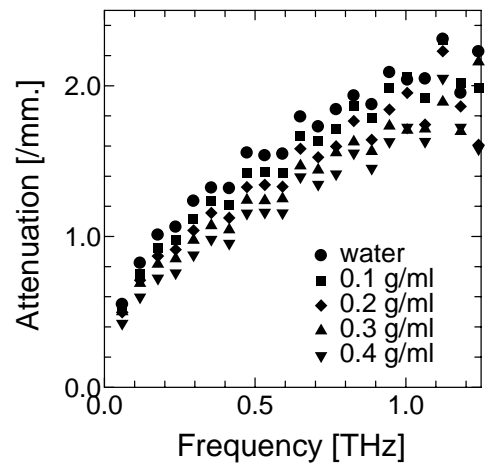

Fig. 6 Change in the attenuation with an immersion of albumin molecules in the water. The attenuation was not converted to the bulk values.

\section{Coplanar Strip Lines}

\subsection{Propagation Characteristics}

Fig. 7 shows the time-domain traces observed in the CPSs of $L=1 \mathrm{~mm}$ on the sapphire, quartz, and polymer substrates [13]. Each trace was normalized by its peak value. Similarly to the case of the MSL shown in Fig. 3, 
ell-resolved two peaks corresponding to the first and second passage of the $\mathrm{THz}$ pulses at the detector were observed in all the devices. However, the decrease in the peak height and the distortion of the pulse shape are obviously larger in the sapphire CPS in comparison with the polymer CPS, indicating the higher attenuation and larger dispersion in the CPS on the substrate of high permittivity.

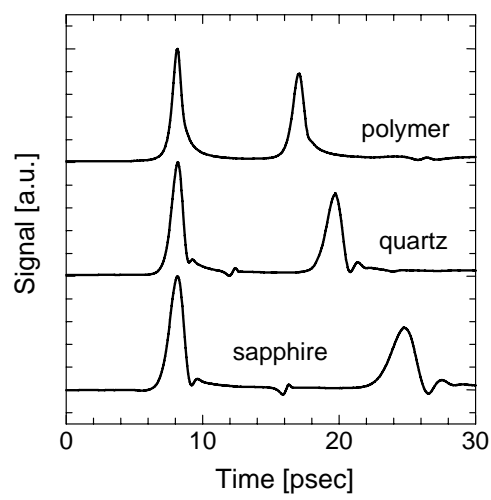

Fig. 7 Examples of the time-domain trace observed in the CPSs made on the polymer, quartz, and sapphire substrates. The distance $L$ between the detector and the end of the line is $1 \mathrm{~mm}$. Each trace was normalized by its peak value and shifted vertically for clarity.

The attenuation and the effective index of refraction were plotted as functions of frequency by the filled symbols in Fig. 8. In comparison with the sapphire CPS, of which the permittivity of the substrate is close to that of the semiconductors such as GaAs and Si widely used in the previous experiments, the attenuation in the polymer CPS is an order of magnitude smaller. The experimental results are qualitatively consistent with the predictions by the FDTD simulation shown by the open symbols. In particular, the agreement is very good in the polymer CPS. The reason for the rather large discrepancy in the quartz CPS is not clear at present. Depicted by the lines are the prediction based on the formulas for the attenuation due to the conductor [18] and radiation [19] losses. The attenuation in the high frequency regime, $f>0.3,0.5$, and $1 \mathrm{THz}$ in the sapphire, quarts, and polymer CPSs, respectively, is dominated by the radiation loss, while it is done by the conductor loss in the low frequency regime. It is obvious that the radiation loss is suppressed by the use of low permittivity substrate. In addition, the conductor loss is also low in the low permittivity CPS, which is because of the high characteristic impedance of the transmission line. As a result of these, the attenuation as low as $0.2 \mathrm{~mm}^{-1}$ was realized in the polymer CPS, and the spectral range reaching $3 \mathrm{THz}$ was achieved as shown in Fig. 9. Another important information may be that the analytical formulas used in microwave regime can predict the attenuation, particularly for the CPS on the low permittivity substrate. The deviation of the prediction based on the analytical model from the FDTD results comes mostly from that in the characteristic impedance, or equivalently the effective dielectric constant as seen in Fig. 8(b).
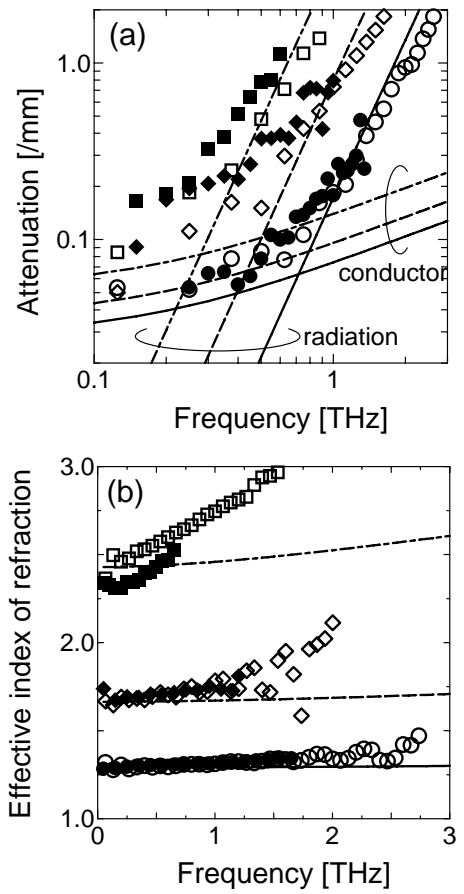

Fig. 8 (a) Attenuation constant and (b) effective index of refraction associated with the propagation of the $\mathrm{THz}$ pulses along the CPS. The filled and open symbols represent the experimental and the FDTD results, respectively. The solid lines are the prediction based on the analytical formulae used in microwave regime.

In the effective index of refraction shown in Fig. 8(b), a very small dispersion is observed in the polymer CPS. The FDTD simulation also explains rather well the experimental results, particularly in the polymer CPS. Also noted is that both the FDTD and experimental results are well predicted by the analytical formula [20] for the low-permittivity CPS. The deviation tends to be large in the CPS on the high permittivity substrates, indicating that the analytical model is not suitable. One of the big differences between the model and the present case is the type of the excitation, i.e. continuous wave or very short pulse. Indeed, for example, the propagation characteristics are dependent on the substrate thickness in the model, while no clear difference was found in the FDTD simulation on the present experiments.

\subsection{Spectroscopy of Powder Specimen}

As a test of a spectroscopic application of the polymer CPS, we measured the attenuation due to the presence of powder specimens. In the measurement, a powder specimen was placed on the lines and covered by another polymer plate, which was pressed by a screw to enhance and stabilize the interaction between the specimen and the $\mathrm{THz}$ field. Examples of the amplitude spectra were shown in Fig. 9 for the case of biotin. The absorption lines are clearly observed up to $2 \mathrm{THz}$ at the frequencies which agree quite well with a reported [21], indicating that the low permittivity polymer CPS can be used as a compact head in THz TDS systems. 


\subsection{Three Strip Coplanar Lines}

As shown in Fig. 8(a), the attenuation in the high frequency regime $f>1 \mathrm{THz}$ increases rapidly due to the radiation loss even in the polymer CPS. To expand the usable frequency range, a further effort to reduce the radiation loss is necessary. One of a possible way is to use the quadrupole field mode on TSCPSs originally proposed in 1997 [22]. In their work, though the suppression of the radiation was clearly demonstrated, the attenuation was not very low since a silicon substrate was used. Hence, it may be promising to use the quadrupole mode with our low permittivity substrate.

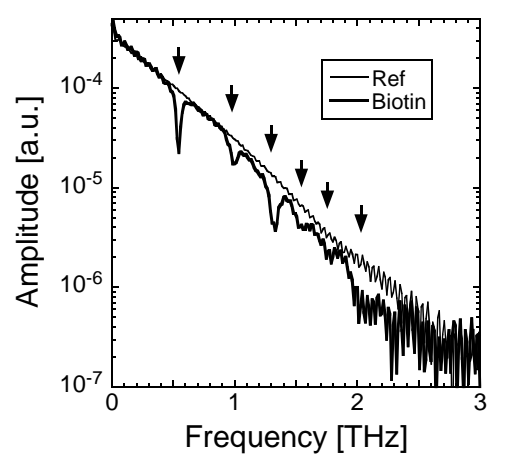

Fig. 9 Amplitude spectra observed in the polymer CPS with and without the biotin powder on the lines. The arrows indicate the positions of the absorption lines reported in the literature.

In the TSCPS device as shown in Fig. 1(c), by changing the polarity of the bias applied to the three lines, both the dipole and quadrupole modes can be excited [14]. Shown in Fig. 10 are the attenuation constant and the effective index of refraction evaluated experimentally. In the dipole mode both on the polymer and quartz TSCPS (open symbols), the frequency dependence is nearly the same as in the two-line CPS, shown in Fig. 8(a). The attenuation is dominated by the conductor loss in the low frequency regime and by the radiation loss in the high frequency regime, with the values higher in the quartz TSCPS than in the polymer one. Obviously the attenuation is lower in the quadrupole mode both in the polymer and quartz TSCPSs in the frequency range where the radiation is the dominant loss in the dipole mode. As expected, both the radiation and conductor losses are suppressed by the use of the low permittivity substrate also in the quadupole mode. However, the experimental values are higher than those of the FDTD simulation shown by the triangles. Though the reason of the discrepancy is not clear at present, it could be due to the asymmetry of the quadrupolar field distribution caused by the imbalance between in the photo-excitation of the two PC switches of the $\mathrm{THz}$ generator. In comparison with the results shown in Fig. 8, the attenuation due to the radiation in the dipole mode is higher in the TSCPS. This is because the total width of the lines corresponding to the dipole length is larger $(100 \mu \mathrm{m})$ in the TSCPS than in the CPS $(60 \mu \mathrm{m})$. The same effect can be expected also in the TSCPSs.
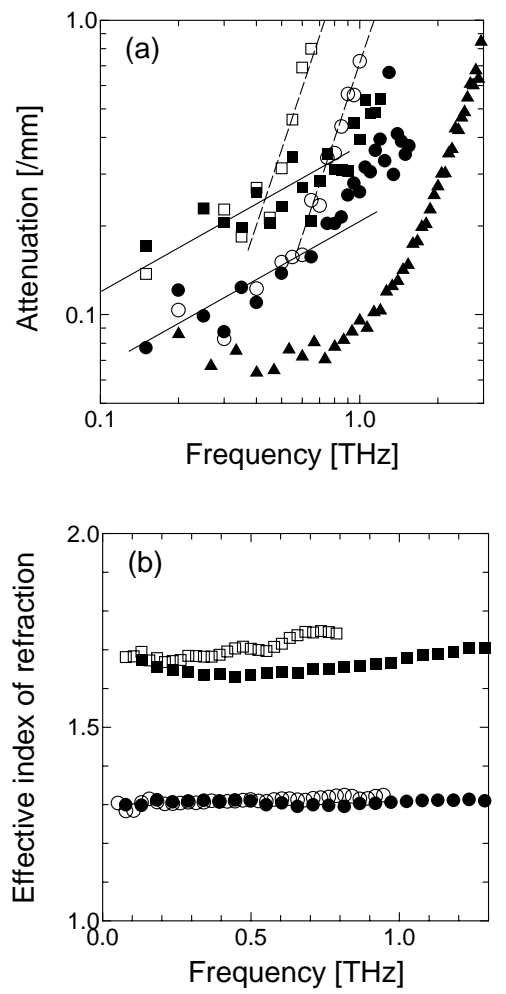

Fig. 10 (a) Attenuation constant and (b) effective index of refraction associated with the propagation of the $\mathrm{THz}$ pulses along the TSCPSs. The filled and open circles are the results of the quadrupole and dipole modes on the polymer TSCPS, and the filled and open squares are those of the quadrupole and dipole modes on the quartz TSCPS, respectively. In (a), the solid and broken lines represent the $f^{1 / 2}$ and $f^{3}$ dependencies respectively, and the filled triangles denote the result of the FDTD simulation.

Hence, optimizations of the device design as well as the proper excitation are expected to lead to a very low attenuation in the $\mathrm{THz}$ range. The dispersion is also smaller in the quadrupole modes both in the quartz and polymer TSCPSs. In particular, no variation was observed within the experimental precision in the measured frequency range.

\section{Summary}

We have investigated the propagation of $\mathrm{THz}$ electrical pulses on the micro-strip lines (MSLs) and coplanar strip-lines (CPSs) utilizing low permittivity polymer materials as the insulator or the substrate. In both types of the devices, THz pulses whose spectra reach $3 \mathrm{THz}$ can be guided in the propagation as long as $1 \mathrm{~mm}$. It was demonstrated that the devices can be used as compact spectroscopic sensor heads for liquid or powder specimens. In the present MSLs, the attenuation was found to be larger than the predictions taking only the conductor loss into account, most likely due to the dielectric loss in the polymer insulator. In contrast, the attenuation in the CPSs was explained quite well by the conductor loss in the low frequency regime, and by the radiation loss in the high frequency 
regime. The dispersion is very small in both types of the devices. The attenuation can be reduced by a use of the materials of lower loss in the MSLs and by the use of quadrupole modes in the CPSs, as partly shown in this work. With such improvements, the strip-line devices are expected to be a powerful tool in the future $\mathrm{THz}$ technology.

\section{Acknowledgements}

The authors acknowledge Dr. I. Hosako of National Institute of Information and Communication Technology, Japan for the TDS measurement of the substrates. This work was supported by the Strategic Information and Communications R\&D Promotion Program of the Ministry of Internal Affairs and Communications, Japan and partly by a Grant-in-Aid for Young-Scientists B from the Ministry of Education, Culture, Sports, Science and Technology, Japan.

\section{References}

[1] AUSTON, D. H., SMITH, P. R. Picosecond optical electronic sampling: Characterization of high-speed photodetectors. Applied Physics Letters, 1982, vol. 41, no. 7, p. $599-601$.

[2] VAldmanis, J. A., MOUROU, G. A., GABEL, C. W. Subpicosecond Electrical Sampling. IEEE Journal of Quantum Electronics, 1983, vol. 19, no. 4, p. $664-667$.

[3] AUSTON, D. H. Impulse response of photoconductors in transmission lines. IEEE Journal of Quantum Electronics, 1983, vol. 19 , no. 4 , p. $639-648$

[4] MOUROU, G. A., MEYER, K. E. Subpicosecond electro-optic sampling using coplanar strip transmission lines. Applied Physics Letters, 1984, vol. 45 no. 5, p. $492-495$.

[5] KETCHEN, M. B., GRISCHKOWSKY, D., CHEN, T. C., CHI, C-C., DULING, III, I. N., HALAS, N. J., HALBOUT, J-M., KASH, J. A., LI, G. P. Generation of subpicosecond electrical pulses on coplanar transmission lines. Applied Physics Letters, 1986, vol. 48, no. 12, p. $751-753$.

[6] SPIRK, R., DULING, III, I. N., CHI, C-C., GRISCHKOWSKY, D. Far infrared spectroscopy with subpicosecond electrical pulses on transmission lines. Applied Physics Letters, 1987, vol. 51, no. 7, p. $548-550$.

[7] GRISCHKOWSKY, D., DULING, III, I. N., CHEN, T. C., CHI, C-C. Electromagnetic shock waves from transmission lines. Physical Review Letters, 1987, vol. 59, no. 15, p. 1663 - 1666.

[8] CHENG, H-J., WHITAKER, J. F., WELLER, T. M., KATEHI, L. P. B. Terahertz-bandwidth characteristics of coplanar transmission lines on low permittivity substrates. IEEE Transactions on Microwave Theory and Techniques, 1994, vol. 42, no. 12, p. 2399 - 2406.

[9] Heiliger, H.-M., NAGEL, M., ROSKOS, H. G., KURZ, H. SCHNIEDER, F., HEINRICH, W., HEY, R., PLOOG, K., Lowdispersion thin-film microstrip lines with cyclotene (benzocyclobutene) as dielectric medium. Applied Physics Letters, 1997, vol. 70 , no. 17 , p. $2233-2235$

[10] NAGEL, M. BOLIVAR, P. H., BRUCHERSEIFER, M., KURZ, H., BOSSERHOFF, A., BUTTNER, R., Integrated THz technology for label-free genetic diagnostics. Applied Physics Letters, 2002, vol. 80, no. 1 , p. $154-156$.
[11] OKUBO, T., ONUMA, M., KITAGAWA, J., KADOYA, Y. Microstrip-line-based sensing chips for characterization of polar liquids in terahertz regime. Applied Physics Letters, 2006, vol. 88, no. 21, article no. 212511.

[12] KITAGAWA, J., OHKUBO, T., ONUMA, M., KADOYA, Y. THz spectroscopic characterization of biomolecule/water systems by compact sensor chips. Applied Physics Letters, 2006, vol. 89, no. 4, article no. 041114.

[13] YANAGI, S., ONUMA, M., KITAGAWA, J., KADOYA, Y. Propagation of terahertz pulses on coplanar strip-lines on low permittivity substrates and a spectroscopy application. Applied Physics Express, 2008, vol. 1, no. 1, article no. 012009.

[14] GUPTA, K. C., GARG, R., BAHL, I., BHARTIA, P. Microstrip Lines and Slotlines. $2^{\text {nd }}$ ed. Norwood: Artech House, 1996, p.108.

[15] KOBAYASHI, M. A dispersion formula satisfying recent requirements in microstrip CAD. IEEE Transactions on Microwave Theory and Techniques, 1988, vol. 36, no. 8, p. 1246 - 1250.

[16] ONUMA, M., OHKUBO, T., KITAGAWA, J., KADOYA, Y., Technical Digest of the 2006 Joint $31^{\text {st }}$ International Conference on Infrared and Millimeter Waves and $14^{\text {th }}$ International Conference on Terahertz Electronics, 2006, p. 466.

[17] LIEBE, H. J., HUFFORD, G. A., MANABE, T. A model for the complex permittivity of water at frequencies below $1 \mathrm{THz}$. International Journal for Infrared and Millimeter Waves, 1991, vol. 12 , no. 7 , p. $659-675$.

[18] CHIONE, G. A CAD-oriented analytical model for the losses of general asymmetric coplanar lines in hybrid and monolithic MICs. IEEE Transactions on Microwave Theory and Techniques, 1993, vol. 41 , no. 9 , p. 1499 - 1510 .

[19] PHATAK, D. S., DAS, N. K., DEFONZO, A. P. Dispersion characteristics of optically excited coplanar striplines: comprehensive full-wave analysis. IEEE Transactions on Microwave Theory and Techniques, 1990, vol. 38, no. 11, p. 1719 - 1730.

[20] HASNAIN, G., DIENES, A., WHINNERY, J. R. Dispersion of picosecond pulses in coplanar transmission lines. IEEE Transactions on Microwave Theory and Techniques, 1986, vol. 34, no. 6, p. 738 741.

[21] KORTER, T. M., PLUSQUELLIC, D. P. Continuous-wave terahertz spectroscopy of biotin: vibrational anharmonicity in the far-infrared. Chemical Physics Letters, 2004, vol. 385, no.1-2, p. 45 - 51.

[22] McGOWAN, R. W., GRISCHKOWSKY, D., MISEWICH, J. A. Demonstrated low radiative loss of a quadupole ultrashort electrical pulse propagated on a three strip coplanar transmission line. Applied Physics Letters, 1997, vol. 71, no. 19, p. 2842 - 2844.

\section{About Authors...}

Yutaka KADOYA was born in Kyoto, Japan, in 1961. He received the B.S. and M.S. degrees in electrical engineering from Kyoto University, Japan in 1984 and 1986, respectively, and the $\mathrm{Ph} . \mathrm{D}$. degree in electrical engineering from the University of Tokyo, Japan in 1996. In 1986, he joined Sumitomo Electric Industries Co. Ltd. where he had been engaged in the development of $\mathrm{GaAs}$ and related semiconductor devices. From 1989 to 1993, he had investigated the ultra-high vacuum processing including molecular beam epitaxy of III-V compounds and the electron transport at the hetero-interfaces in Sakaki Quantum Wave Project, ERATO, the Japan Science and Technology 
Agency. In 1994 he joined Hiroshima University, Higashihiroshima, Japan, as a research associate. In 1998 he was promoted to an associate professor. Since 2004, he has been a full professor in the Department of Quantum Matter, Graduate School of Advanced Science of Matter, Hiroshima University. His research interest includes the optoelectronic as well as quantum-optical phenomena such as the $\mathrm{THz}$ wave emission from exciton-polaritons in quantum wells, semiconductor light emitting diodes generating sub-Poissonian lights, transmission line devices in $\mathrm{THz}$ regime, free space $\mathrm{THz}$ emitters and detectors, and the generation of quantum-entangled photons. He is a member of the Japan Society of Applied Physics, the Physical Society of Japan, the Institute of Electrical and Electronics Engineers, and the Optical Society of America.

Masayuki ONUMA was born in Miyagi, Japan, in 1982. He received the B.S. and M.S. degrees in electronic materials engineering from Hiroshima University, Japan in 2006 and 2008, respectively. In 2008, he left Hiroshima University and joined Canon Inc., Japan.

Tetsuya OHKUBO was born in Yamaguchi, Japan, in 1981. He received the B.S. and M.S. degrees in electrical engineering from Hiroshima University, Japan in 2004 and 2006, respectively. In 2006, he left Hiroshima University and joined Kyocera Corp., Japan.
Shinji YANAGI was born in Nagasaki, Japan, in 1984. He received the B.S. degree in electronic materials engineering from Hiroshima University, Japan in 2007. In 2007, he left Hiroshima University and joined Elpida Memory Inc., Japan.

Naoko SATO was born in Fukuoka, Japan, in 1984. She received the B.S. degree in electronic materials engineering from Hiroshima University, Japan in 2008. Since 2008, she has been a graduate student of Hiroshima University, Japan.

Jiro KITAGAWA was born in Osaka, Japan, in 1970. He received the B.S. degree in physics from Kyoto University, Japan in 1993, and the M.S. and Ph.D. degrees in physics from the University of Tokyo, Japan in 1995 and 1998, respectively. From 1998 to 2001, he was a research fellow of the Japan Society for the Promotion of Science. In 2002 he joined Hiroshima University, Higashihiroshima, Japan, as a research associate. Since then, he has been engaged in the study of material properties using the $\mathrm{THz}$ time-domain spectroscopic method and the development of $\mathrm{THz}$ devices. His current research interests are $\mathrm{THz}$ dynamics in magnetic compounds and semiconductors, $\mathrm{THz}$ spectroscopic chips using solid state transmission lines and free space $\mathrm{THz}$ emitters and detectors. He is a member of the Japan Society of Applied Physics and the Physical Society of Japan.

\title{
Correction to: Planar Tri-Band Antenna Design
}

\author{
Michal POKORNÝ, Jiři HORÁK, Zbyněk RAIDA \\ Dept. of Radio Electronics, Brno University of Technology, Purkyňova 118, 61200 Brno, Czech Republic \\ xpokor33@stud.feec.vutbr.cz,xhorak23@stud.feec.vutbr.cz, raida@feec.vutbr.cz
}

In the above-mentioned paper in Radioengineering Journal, vol. 17, no. 1, April 2008, page 36, an error occurred. The correct reference to [1] is the following:

[1] CIAIS, P., STARAJ, R., KOSSIAVAS, G., LUXEY, C. Design of an internal quad-band antenna for mobile phones. IEEE Microwave and Wireless Components Letters, April 2004, Vol. 14, no. 4, p. 148 - 150.

Thanks to all who have notified the authors of errors in the text. 